\title{
ON A PRACTICAL WAY OF DESCRIBING FORMAL DEDUCTIONS
}

\author{
KATUZI ONO
}

\section{Introduction}

Even though the logical structure of any formal deduction can be nicely expressed in a tree-form diagram, it is more practical to write it down in a series of propositions. In each step of inference, we usually deduce a proposition on basis of some foregoing propositions". However, global aspects of mathematical theories show us that this is not always the case. For, in mathematical theories, theorems are usually stated before their proofs. In fact, also in proofs of theorems, it is often practical that we prove propositions after stating them. Accordingly, in our real way of thinking, we arrange propositions going back and forth in the logical order.

In formal deductions, some propositions are stated as temporary assumptions, definitions, or something like that. Furthermore, propositions such as "Take any object, say $x$, satisfying the conditions $₫(x)$." nominate temporarily the variable $x$, having the assumption character. On the other hand, propositions such as "There is an object $t$ satisfying the condition $\Subset(t)$, so take any one of such objects and call it $x$." also nominate temporarily the variable $x$, having the assertion character. In such cases, we are tempted to use the universal or existential quantifiers, though confusions are hardly avoidable without introducing some devices.

In Section (1), we introduce new symbols of the forms $\forall x$ ! and $\exists x$ ! called nominating quantifiers to meet the demand. Namely, by making use of these quantifiers, we denote the former example as " $\forall x !(\varsigma(x) "$ and the latter one as " $\exists x !(5(x)$ ".

In Section (2), we introduce a system of numbering of all the propositions in any formal deduction. We use Latin letters for numbering propositions in

Recieved May 4, 1962.

1) Such is the case for the most of logical systems, e.g. Gentzen's systems [1]. Kuroda [2] introduced a system, in which inferences are described in the inverse direction. 
a formal deduction which is usually a proof of a theorem, as it looks advisable to reserve numerals for numbering theorems and definitions.

Our system is properly designed so as to fulfill the following four conditions :

A. Propositions are arranged in the lexicographical order of their numbers. (Check this in the example of Section (3).)

B. One can see easily only by numbers, whether any proposition is qualified to be a logical basis of a given proposition. (Just behind every proposition, we indicate its logical basis by numbers following after the symbol "/". In the example of Section (3), the propositions $\mathbf{A}-\mathbf{j}$, ka, $\mathbf{k b A}, \mathbf{k b b A}-\mathbf{k b b e}$ are qualified to be basis of $\mathbf{k b b}$, the propositions $\mathbf{A}-\mathbf{j}, \mathbf{k a}, \mathbf{k b \mathbf { A }}, \mathbf{k b b}, \mathbf{k b c}, \mathbf{k b d}, \mathbf{k b e}$ are qualified to be basis of $\mathbf{k b}$, and the propositions $\mathbf{A}-\mathbf{j}, \mathbf{k a}, \mathbf{k b}$ are qualified to be basis of $\mathbf{k}$.)

C. For any one of temporary assumptions, definitions, and nominations, one can easily see only by numbers its scope in which it is valid. (In the example of Section (3), all the propositions from kac to kae i.e. the propositions kac, kacA-kacc, kad, kadA-kadc, kae are in the scope of the nomination kab).

D. For any propositon stated before its proof in the deduction, one can easily see only by numbers, what part of the deduction is devoted to the proof of the proposition. (In the example of Section (3), all the propositions from kbA to kbe i.e. the propositions kbA, kbb, kbbA-kbbe, kbc, kbcA-kbee, kbd, kbe form a proof of $\mathbf{k b}$ ).

In Section (3), we explain our way of description by an example which asserts that a special case of the axiom of replacement of the Fraenkel settheory implies that there is no set containing all the sets as members. Although it is sometimes desirable to express the leading idea of a formal deduction more vividly by an illustration in ordinary sentences, our way of description may be recognized by this example as being practical enough to take notes of mathematical proofs without being too long and always exposing their logical structure exactly.

\section{(1) Nominating quantifiers}

Let us express by $Q_{i}$ either of the quantifiers $\forall$ or $\exists$. Symbols of the form $Q_{1} x_{1} \cdots Q_{n} x_{n}$ ! are called nominating quantifiers, assuming that $x_{1}, \ldots, x_{n}$ 
are all distinct. Nominating quantifiers can stand only at the top of propositions. Such propositions are called nominations of the variables $x_{1}, \ldots, x_{n}$.


and $\exists x$ ! $(x)$ means "There is an object $t$ satisfying the condition $\S(t)$, so take any one of such objects and call it $x$ ". Propositions of this kind are called simple nominations.

General nomination of objects of the form $Q_{1} x_{1} \ldots Q_{n} x_{n} !\left(s\left(x_{1}, \ldots, x_{n}\right)^{2}\right)$. is an abbreviation of the series of the following simple nominations:

$$
\begin{gathered}
Q_{1} x_{1} !\left[Q_{2} x_{2} \ldots Q_{n} x_{n} \cdot \Subset\left(x_{1}, \ldots, x_{n}\right)\right]_{1}, \\
\cdots \\
Q_{n-1} x_{n-1} !\left[Q_{n} x_{n} \cdot\left(5\left(x_{1}, \ldots, x_{n}\right)\right]_{n-1},\right. \\
Q_{n} x_{n} ! \Subset\left(x_{1}, \ldots, x_{n}\right),
\end{gathered}
$$

where $[\mathfrak{U}]_{k}$ denotes $\mathfrak{I} \rightarrow \mathfrak{I}$ (a true proposition containing no free variables) or $\mathfrak{U}$ according as $Q_{k}$ denotes $\forall$ or $\exists$.

In any formal deduction, all the propositions which are qualified to be basis (defined in Section (2)) of a nomination of variables, say $x_{1}, \ldots, x_{n}$, should stand before the nomination in the lexicographical order, and these variables $x_{1}, \ldots$, $x_{n}$ should not occur in them as free variables ${ }^{3)}$. Any nominating quantifier nominates free variables temporarily in a part of the deduction called the scope (defined in Section (2)) of the nomination. In the scope of the nomination, these variables are regarded as free.

\section{(2) Numbering}

To every proposition in a formal deduction, we give a number i.e. a finite sequence of letters, any one of which except the last letter of the number of any temporary assumption, definition, or something like that is a small letter. If necessary, we supply a series of letters $\overline{\mathbf{a}}, \overline{\mathbf{b}}, \ldots$ coming after the letter $\mathbf{z}$, and also $\overline{\overline{\mathbf{a}}}, \overline{\overline{\mathbf{b}}}, \ldots$ coming after the letter $\overline{\mathbf{z}}$, and so on. The same also for the capital letters standing at the end of the numbers of any temporary as-

2) Sometimes it is convenient to denote propositions of the form $Q_{1} x_{1} \cdots Q_{n} x_{n} !(\mathfrak{I} \rightarrow \mathfrak{I})$ simply by " $Q_{1} x_{1} \cdots Q_{\|} x_{4} ! "$.

3) We can impose this condition on all the propositions standing before the nomination. To be practical, however, we impose this condition only on propositions qualified to be basis of it. Evidently, this condition corresponds to the so-called variable condition for introducing $\forall$ and $\exists$ in ordinary logical systems. 
sumptions or definitions.

All the propositions in a formal deduction are arranged in the lexicographical order of their numbers. The numbering should be given in the following way: If there is a number of the form $\alpha t$, there are also all the numbers of the forms $\alpha, \alpha \mathbf{a}$ (or $\alpha \mathbf{A}$ ), . . , $\alpha$ t where $\alpha$ denotes a number (including the vacant one) and $t$ denotes a letter. All the propositions having the numbers of the form $\alpha \xi \varphi$ form a proof of $\alpha$. If $\alpha$ is vacant, they form the whole deduction. (Here we use the word "a proof of $\alpha$ " in the sense that it is the whole series of propositions which proves $\alpha$ by making use of any propositions already proved before it).

Any proposition $\alpha$ is qualified to be a basis of another proposition $\beta$, if $\alpha$ is a number of the form $\beta$, or if $\alpha$ and $\beta$ are numbers of the forms $\gamma$ and $\gamma t \rho$ respectively and $\xi$ stands before $t$ in the alphabetical order. Notice that $\gamma \xi \theta$ is not qualified to be a basis of $\gamma+\varphi$ unless $\theta$ is vacant. In other words, the logical order is not transtive. This may sound strange, but it is rather natural if we take into account that all the propositions having the numbers of the form $\gamma$ st $\lambda$ form a proof of $\gamma$ s.

If $\alpha$ is qualified to be a basis of $\beta$ and also stands before $\beta$ in the lexicographical order, we say that $\beta$ is in the scope of $\alpha$. Temporary assumptions, definitions, and nominations are valid only in their scopes.

Any proposition having a number ending with a capital letter is a temporary assumption, definition, or something of that kind ${ }^{4}$. It is not a conclusion of an inference anyway, so we do not have to indicate its basis.

Any other proposition is a conclusion of an inference. Usually, they are deduced from some premises. We indicate these premises by their numbers just behind the proposition following after the symbol "/" (read "by"). They are theorems already proved, definitions, or propositions qualified to be basis of these propositions. If the proposition is proved by the propositions following after it, its number is marked by “))". (Otherwise, marked by “)”.)

\section{(3) Example}

To show an example of a proof described in our way, we introduce an

4) Notice that nominations are not always propositions of this kind. Indeed, nomina-




operator " $\{$ )" (mapping binary relations to binary relations). Namely, $x\{I$ ')y means $\forall t(t \in x \equiv t \Gamma y)$, where $\Gamma$ denotes any binary relation. Evidently, $x\{\Gamma) y$ is unique regarding it as a mapping $y$ to $x$, if we assume the axiom of extensionality. Accordingly,

$$
\forall m \exists p \cdot p\{\{\Gamma\rangle \in) m^{5)}
$$

can be regarded as a special case of the axiom of replacement.

By the assumption $\forall m \exists p \cdot p\{\{\Gamma) \in) m$ only, we can conclude $>\forall x \cdot x \in z^{6}$. In the following, we show a formal proof of this theorem in our way of description.

Proof /reductio ad absurdum: $\mathbf{A} \rightarrow \mathrm{t}^{7}$.
A) Assume $\forall z \exists p \cdot p\{\{\Gamma) \in) z$ and $\exists m ! \forall t \cdot t \in m^{8)}$.
B) Define “" " by $x=y \cdot \stackrel{d f}{\equiv} \forall s(s \in x \equiv s \in y)$.
c) $\forall x \cdot x=x / \mathrm{B}$.
d) $\forall x y(x \in=y \cdot \rightarrow x \in y)$ /B.
E) Define $\Gamma$ by $x \Gamma y \cdot \stackrel{d f}{=}(x \in y \wedge y \notin y)$.
f) $\exists p ! p\left\{\left\{I^{\prime}\right) \in\right)_{m} /$ A. $\quad$ g) $\forall u\left(u \in p \equiv \exists z\left(u\langle\Gamma)_{z} \wedge z \in m\right)\right) \quad$ /f.
h) $\forall u(\exists z(u\{\Gamma) z \wedge z \in m) \equiv: \exists z \cdot u\{\Gamma) z) \quad /$ A.
i) $\forall u\left(u \in p \equiv: \exists z \cdot u\left\{I^{\prime}\right) z\right) / g, \mathbf{h}$.
j) $\forall u(u \in p \equiv \exists z \forall t(t \in u \equiv \cdot t \in z \wedge z \notin z)) \quad / \mathbf{i}, \mathbf{E}$.

k)) $\forall u(u \in p \equiv \cdot \exists z(u=z \wedge z \notin z) \vee(\exists z \cdot z \in z: \wedge: \forall t \cdot t \notin u)) \quad / \mathbf{k a}, \mathbf{k b}^{9)}$.

ka)) $\forall u(u \in p \rightarrow \cdot \exists z(u=z \wedge z \notin z) \vee(\exists z \cdot z \in z: \wedge: \forall t \cdot t \notin u))$

$$
/ \mathbf{k a A} \rightarrow \mathbf{k a e}^{10)} \text {. }
$$

5) In our new object theory, which will be published in the near future, $\exists P \cdot P(\{\Gamma) \sigma) M$ is the only axiom scheme, where $\sigma$ is a binary relation defined by $\epsilon$, the only primitive notion of the system. The relation $\sigma$ is slightly deviated from the satellite notion of our former object theory [3]. However, it is defined in such a way that $\forall X Y(X \in Y$ $\rightarrow X \sigma Y$ ) holds. Our former system is, so to say, an object theory in the manner of the Zermelo set-theory, whereas our new system can be regarded as an object theory in the manner of the Fraenkel set-theory.

6) The proof of this example is a deformation of the Russell paradox. This simple example is one of the clues which lead to our new object theory. Indeed, one can conclude easily by this example proof that $\forall M \exists P \cdot P\left\{\left(\Gamma^{\prime}\right) \sigma\right) M$ implies $\rightarrow \forall X \cdot X \in Z$, if we assume $\forall X Y(X \in Y \rightarrow X \sigma Y)$.

7) This means that the whole deduction from $\mathbf{A}$ to $\mathbf{t}$ forms a proof of this theorem by reductio ad absurdum. (Compare with the proof of 1.)

8) This is an abbreviation of the following two steps. First step: Assume $\exists m \forall t \cdot t \in m$. Second step: $\exists m ! \forall t \cdot t \in m$ /first step. (Compare with kbbA and kbcA.)

9) This means that $\mathbf{k}$ is proved on the basis of $\mathbf{k a}$ and $\mathbf{k b}$.

10) This means that ka is proved by the whole deduction from kaA to kae (Compare with kac, for example). 
kaA ) $\forall u ! u \in p . \quad$ kab) $\exists w ! \forall t(t \in u \equiv(t \in w \wedge w \notin w)) \quad / \mathbf{k a A}, \mathbf{j}^{(1)}$. kac)) $\quad w \notin w \rightarrow \exists z(u=z \wedge z \notin z) \quad /$ kacA $\rightarrow$ kacc.

kacA) Assume $w \notin w^{12)}$. kacb) $\quad u=w / \mathbf{k a b}, \mathbf{k a c A}, \mathbf{B}$.

kacc) $\exists z(u=z \wedge z \leftarrow z)$ /kacb, kacA.

$\operatorname{kad})) \quad w \in w \rightarrow(\exists z \cdot z \in z \wedge \forall t \cdot t \notin u) \quad / \operatorname{kad} \mathbf{A} \rightarrow \operatorname{kadc}$.

$\operatorname{kadA}) \quad$ Assume $w \in w . \quad \operatorname{kadb}) \quad \forall t \cdot t \notin u / \mathbf{k a b}, \mathbf{k a d A}$.

kadc) $\exists z \cdot z \in z: \wedge: \forall t \cdot t \notin u / \operatorname{kadA}, \mathbf{k a d b}$.

kae) $\exists z(u=z \wedge z \notin z) \vee(\exists z \cdot z \in z: \wedge: \forall t \cdot t \notin u) \quad /$ kac, kad.

kb)) $\exists z(u=z \wedge z \notin z) \vee(\exists z \cdot z \in z: \wedge: \forall t \cdot t \notin u) \cdot \rightarrow u \in p \quad / \mathbf{k b A} \rightarrow \mathbf{k b e}$.

kbA) Assume $\exists z(u=z \wedge z \notin z) \vee(\exists z \cdot z \in z: \wedge: \forall t \cdot t \notin u)$.

kbb)) $\exists z(u=z \wedge z \notin z) \rightarrow \exists z(u\{\Gamma) z \wedge z \in m) \quad /$ kbbA $\rightarrow$ kbbe.

kbbA) Assume $\exists z$ ! $(u=z \vee z \notin z)$.

kbbb) $\forall t(t \in u \equiv t \in z) \quad / \mathbf{k b b A}$.

kbbc) $\quad \forall t(t \in u \equiv(t \in z \wedge z \notin z)) \quad / \mathbf{k b b b}, \mathbf{k b b A}$.

kbbd) $\quad u\{\dot{\Gamma}) z$ /kbbc, E. $\quad$ kbbe $) \quad \exists z(u\langle\Gamma) z \wedge z \in m) \quad$ /kbbd, A.

kbc)) $\quad(\exists z \cdot z \in z: \wedge: \forall t \cdot t \notin u) \rightarrow \exists z\left(u\left\langle I^{\prime}\right) z \wedge z \in m\right) \quad /$ kbcA $\rightarrow$ kbce.

kbcA) Assume $\exists z ! z \in z$ and $\forall t \cdot t \notin u$.

kbcb) $\forall t \cdot \neg(t \in z \wedge z \notin z) \quad / \mathbf{k b c A}, \mathbf{B}$.

kbcc) $\forall t(t \in u \equiv \cdot t \in z \wedge z \notin z) \quad / \mathbf{k b c A}, \mathbf{k b c b}$.

kbed) $\quad u\{\Gamma) z$ /kbec, E. kbce) $\exists z(u\{\Gamma) z \wedge z \in m) \quad$ /kbed, A.

kbd) $\exists z\left(u\left\langle I^{*}\right) z \wedge z \in m\right) \quad$ /kbA, kbb, kbc kbe $u \in p$ /kbd, g.

l) ) $p \in p \quad$ /reductio ad absurdum: $\mathbf{1 A} \rightarrow \mathbf{l d}$. IA) Assume $p \notin p$.

lb) $p=p \wedge p \notin p \quad$ /c, lA. lc $\exists z(p=z \wedge z \notin z) /$ lb.

Id) $p \in p / \mathbf{k}, \mathbf{l c}$.

m) $\exists t \cdot t \in p / \mathbf{l}$ n) $\exists q !(p=q \wedge q \notin q) / \mathbf{k}, \mathbf{l}, \mathbf{m}$.

o) $\quad q=q \wedge q \notin q$ /c, n. $\quad$ p) $\quad \exists z(q=z \wedge z \notin z) \quad /$.

q) $q \in p / \mathbf{k}, \mathbf{p}$. r) $\quad q \in=q / \mathbf{q}, \mathbf{n} . \quad$ s) $\quad q \in q / \mathbf{r}, \mathbf{d}$.

t) Contradiction $/ \mathbf{n}$, s.

11) This nomination is valid until kae.

12) The assumption is valid until kacc. 


\section{References}

[1] G. Gentzen, Untersuchungen über das logischen Schliessen. Math. Z., 39, pp. 176210, 405-431 (1935).

[2] S. Kuroda, An investigation on the logical structure of mathematics (I). Hamburger Abhandlungen, 22, pp. 242-266 (1958).

[ 3 ] K. Ono, A theory of mathematical objects as a prototype of set theory. Nagoya J. of math., vol. 20 (in press).

Mathematical Institute,

Nagoya University. 